\title{
Del quiebre individual al quiebre histórico: una experiencia de trabajo con víctimas de la represión política

\author{
Dr.Jaime Landa ${ }^{1}$
}

El Instituto Latinoamericano de Salud Mentaly Derechos Humanos (LAS) fue creado en el año 1988 por ungrupodeprofesionales, quese desempeñanenelámbito dela SaludMental y los Derechos Humanos desde elaño1980.

Apesardeltiempotranscurrido, 10años desdesu fundación y 9 años de gobiernos constitucionalmente constituidos, lademandadeatenciónseincrementapermanentementeyaseaporfaltadeapoyoterapéutico previoodebidoalavulnerabilidadpropiadeestoscasos para enfrentarcrisis evolutivas, inserción laboral ode otrana- turaleza. Particularmente relevante es el impacto en la segunday tercerageneración, loque se expresaenconsultas solicitadas muchotiempo después de ocurridoel hecho represivo. LASha desarrollado múltiples actividades de investigación vinculadas tanto al área clínica comoalárea psicosocial, en un permanente intento de relacionarlos problemas individuales con los aspectos macrosocialesasociados.

El trabajoque voy a presentar, ocupa material clínico deprocesosterapéuticos, auyos nombres y datos han sido modificados.

\section{El Quiebre Individual}

El golpe militar en Chile, que sucedió el 11 de Septiembre de 1973, es una situación que Tomás Moulian la describe sintéticamente como «el sangriento parto de este Chile actual $\eta^{2}$. El gobierno constitucional de Salvador Allende G. no alcanza a cumplir 1.000 dias y luego de un período de sabotaje y boicot de parte de grupos económicos produjo la cruenta intervención de las fuerzas armadas. Según el informe Rettig consigna con 3.200 muertos mas 650 que refiere la Corporación y algunos casos identificados posteriormente, hace que la cifra de 4.000 desaparecidos sea casi exacta respecto a los casos notificados.

El 11 de Septiembre de 1973 se produce para Rosa un cambio radical en su vida, todo lo que hasta ese momento era seguro deja de serlo, fue como si una bomba hubiera estallado en su vida, fragmentándola en mil pedazos. La imagen del bombardeo de la Moneda, sede del gobierno constitucional, fragmenta otro orden, el orden constitucional del pais, esta es otra bomba. La vida de Rosa se quiebra, la vida del país se quiebra, ¿`son quiebres diferentes?, ¿es uno producto del otro?.

Rosa en la primera consulta que hace a nuestrainstitución, intenta poderarticular un discurso que le permita dar cuenta de lo que le ha pasado, es posible darse cuenta que, varios años después de ocurrido el momento de su detención, esto aún no es posible.

Rosa viene como habitualmente lo está haciendo desde hace dos meses, una veza la semana, se sienta 
y nuevamente me reitera que no sabe para que le sirve venir, dice «todo esto que le cuento es algo que me pasóy no saco nada con hablar, solo me da pena". En el momento que consulta tiene 65 años, su experiencia traumática le ocurre cuando tiene 40 años, su vida en ese momento, ya estaba encaminada, tenia cuatro hijos y dos nietas, su marido profesional universitario tenia un buen trabajo, ella se dedicaba a las labores de la casa. El 13 de Septiembre en la noche es detenida, separada de su familia y no vuelve más a su casa, permanece detenida por tres meses, sale al exilio junto con su familia a la que vuelve a ver sólo en el momento en que toma el avión que la lleva fuera de Chile, "eso me cambió la vida, yo no podia quebrarme, no podia llorar, tenia que mantenerlos a todos juntos, tuve que seguir». Hay un cambio radical en su vida, comienza un largo deambular por diferentes paises, para finalmente quedarse en Europa, vuelve hace unos tres años, consulta en nuestra institución a instancias de sus hijos, dos de ellas son tratadas acá.

Este día Rosa esta algo más apesadumbrada, pero también rabiosa, "el médico me dijo que la colitis ulcerosa es una enfermedad que no se me va a quitar, yo nunca pensé que era una enfermedad crónica, pensaba que me iba a mejorar un poco pero no que se iba a mantener siempre, el Dr. dijo que por eso tengo este problema a la vista (conjuntivitis), y que además también debe ser la causa de la reacción alérgica al audífono, me lo pongo y me produce dolor, por eso no lo puedo usar". Rosa está con una disminución auditiva importante, ve con alguna dificultad, pero ha venido a la hora como lo ha hecho en todo este tiempo. Pienso si esa enfermedad crónica de la que habla, también puede ser este duelo que no ha podido elaborar. Hay una laceración equivalente a la que tiene en el intestino.

Me tengo que acercar mucho para poder hablar sin gritarle, ya que su audífono está inutilizado. Se produce un clima de intimidad, la cercania me permite ver con claridad su rostro, que si bien lo habia visto durante este tiempo, no con el detalle que podia verle ahora, sus ojos son de un azul muy claro, hay silencios largos pero no son incómodos. Ella vuelve a reiterar que no sabe para que viene, me vuelve a preguntar si esto le serviria.

\section{ElEspacioTerapéutico:EspaciodelaReparación}

En su historia el quiebre ha producido un vacio, que Rosa ha intentado resolver a través de la omnipotencia primitiva ${ }^{3}$, que es un modo de sobrevivir psiquicamente a la experiencia de no integración en ausencia de un objeto que pueda contener tal estado. Jordán ${ }^{4}$ señala además que es una defensa, que permite la sobre vida en situaciones de extrema ansiedad. Estas se producen durante el desarrollo cuando falla el objeto en su función de proveer una envoltura psiquica en la cual puedan contenerse los aspectos desintegrados de la personalidad, función que luego es internalizada concretamente en la piel. Cuando esta función falla, el sujeto expe- rimenta la sensación de tener un hoyo en la piel a través del cual se escurre toda la vida psiquica, señala además que la omnipotencia primitiva en situaciones de persecución politica junto a otros mecanismos de defensa constituye los mecanismos de sobre vivencia psiquica.

¿Qué es lo que Rosa pregunta?, ¿a qué apunta su pregunta?, en un primer plano es saber si tanta pena le sera útil, si serviria. En otro plano tal vez más profundo, saber si podrá unir lo que fue su vida pasada con esta experiencia que tiene ahora al volver a su patria, juntar lo que se ha quebrado. En el espacio terapéutico se produce nuevamente

\footnotetext{
${ }^{3}$ Joan Symngton, 1985.

${ }^{4}$ Juan Francisco Jordán. Una experiencia en supervisión de orientación psicoanalítica con especial énfasis en las fantasías y sentido de omnipotencia. 1997, (no publicado)
} 
esa sensación de impotencia que ha tenido Rosa por todos estos años. Becker ${ }^{5}$ señala... «la disposición del terapeuta de conectarse con esta «agonía de angustia", conteniéndola, ofreciéndola un espacio auténtico pero suficientemente seguro para que el paciente puede intentar una integración. $\mathrm{O}$ dicho de otra manera, el terapeuta debe permitir el surgimiento de sus propios potenciales fragmentos manteniendo su disposición a la contención para facilitar al paciente el reconocimiento de si mismo en sus fragmentos lo que implicaría el inicio de una integración".

\section{Traumatización Extrema}

La experiencia de daño es una experiencia que fractura la vida de las personas, se produce una separación entre los acontecimientos ocurridos antes y después de ocurrida esta situación. De forma tal que la vida deja de tener una estructura con sentido, o por lo menos este sentido sufre una suerte de desasimiento, esta tensión que organizaba la vida, se afloja, es posible interpretar esta sensación y la sintomatologia que produce como una depresión que sin lugar a dudas lo es pero en donde claramente hay algo más.

Cuando usamos el concepto de stress posttraumático para definir esta situación, vemos que este es insuficiente, nos remite a la descripción de la sintomatología principalmente, y la referencia al trauma es vaga, de esa forma una catástrofe natural, un accidente automovilistico, un ataque al corazón, la detención y tortura posterior, constituyen experiencias no significativamente diferentes ${ }^{6}$. Quedamos sin poder pensar todo el significado del contexto que el trauma tiene.

El término "traumatización extrema" usado en nuestro equipo, enfatiza que se trata de un proceso individual y social que se produce en dependencia de una forma de ejercer el poder en una sociedad, donde las estructuras sociopoliticas se basan en la destrucción y extinción de algunos miembros de la sociedad por otros miembros de la misma sociedad $^{7}$ ", y como agrega coloquialmente D.Becker «es importante reconocer que la traumatización no solo ocurre en la cabeza de las personas sino también entre ellas".
En Rosa este es el periodo de inicio de un proceso terapéutico que será largo y muy costoso, ella viene para demostrar que nada de lo que pueda hacer en la terapia será suficiente, y es posible que en parte tenga razón, pensar lo contrario sería actuar la omnipotencia, antes descrita, en el espacio terapéutico. También sabemos que, teóricamente, este proceso la llevará a elaborar esa pérdida, una vez que se pueda elaborar la rabia que por tanto tiempo ha contenido, lo que no sabemos es como esto será posible. En este sentido lo que yo le respondo a la pregunta que hace Rosa, de si esto le serviría o no, es de que si, de que será algo útil, pero que no sé como esto se dará, que esto es algo que tendremos que averiguar juntos.

Creo que en Rosa se ejemplifica claramente lo que Kinston y Cohen han definido como un hoyo en la mente, una herida persistente, es un espacio que no tiene representación, carece de simbolización.

Citamos a Kinston y Cohen «El analista puede y debe convertirse en parte del objeto primario delanalizando. Sin embargo, para que se produzca crecimiento, elanalista tendrá que establecer un contacto directo con el paciente en estado de represión primaria. Esto parece ser posible únicamente mediante la presencia de una forma especial de relación que denominamos relación primaria... Emplearemos el término "relación primaria" para hacer referencia a la relación de valoración directa, de consideración, confianzay reflejo con otras personas, relación que toda persona necesita de manera absoluta y objetiva. Esta relación se caracteriza por un apego mutuo de carác-

\footnotetext{
${ }^{5}$ Tratamiento de pacientes traumatizados extremos: la importancia del vinculo. ILAS, 1989.

${ }^{6}$ Trauma psicosocial y adolecentes latinoamericanos: Formas de acción grupal. ILAS. Editores David Becker, Germán Morales, María I. Aguilar. Ediciones Chile America CESOC 1994.

${ }^{7}$ Becker D. y Castillo M. I. 1993. "El Tratamiento psicoterapéutico de pacientes traumatizados extremo”, Revista Chilena de Psicoanálisis 10(1).
} 
ter intenso y por una comunicación empática profunda...constituye un complemento ambientaldel individuo que, por ende, no puede ser internalizada. Cuando no existe una forma activa de relación primaria, el individuo vive en un estado de muerte psíquica con el objeto primario.. ${ }^{8}$
En este primer momento con Rosa seguimos la idea de gratificar la nesecidad sin entrar frustrar los deseos, tenemos que constituirnos en el objeto primario del paciente y así facilitar un ambiente un espacio al paciente en el cual pueda creer.

\section{El Quiebre Histórico}

Hacer una relación entre lo que consideraremos como lo individual, pensándolo como lo psicológico, y lo histórico como lo social, es una tarea dificil más aún si lo que veremos tiene relación con el quiebre que se produce en ambos campos y que este quiebre tiene que ver con la experiencia de daño. Lo que pretenderemos es observar como en uno y otro campo los fenómenos que se producen son analogables.

Sabemos que ambos campos, el social y el clinico no pueden ser pensados separadamente, y que se soportan, se contienen mutuamente, su interrelación es fundamental, sobre todo cuando estamos hablando de un tipo de daño que es organizado sistemáticamente como politica de estado durante 17 años. También sabemos que esta pretensión, de hacer una relación entre ambos campos, es inacabable.

El campo clínico al quedar relegado a sólo una de sus funciones, al saber, como un saber técnico, que produce su objetivación más clara en la terapéutica, invalida su reflexión en cualquier otro sector como puede ser el social. De esta forma el saber clínico queda sin poder reflexionar sobre la génesis y mantencion del daño cuando este, en un porcentaje importante, está determinado por lo social, la pregunta sobre la génesis sólo será posible hacerla fuera del espacio de lo que se ha considerado como lo clásicamente clínico, de lo académico, de lo institucional, es decir de los espacios que validan la clinica como un saber integral. Es interesante lo que nos señala G. Deleuze «Los cuerpos enfermos solo son elementos alterados en una maquinaria que piensa en los desperfectos como impedimento para la realización de la tarea" En la dictadura entonces, es dificil que se generen discursos que cuestionen el daño en su génesis, el conocimiento, como vemos está fragmentado, como lo está el cuerpo social, como lo está concretamente el cuerpo del detenido. Quiebre y fragmento palabras que se repiten.

El quiebre político producido en 1973, produce una interrupción al desarrollo social que hasta ese momento se había producido en la historia de Chile. Como todo gobierno dictatorial, define y estructura discursos egemonicos que no pueden ser cuestionados. La característica de gobierno fundacional que se quiere dar al gobierno militar en Chile, promulgación de una nueva constitución - es posible verla como algo que se repite en otras experiencias que como estas se dieron en América en la década del 70 y el 80 .

En el libro Psicología de la Amenaza política y el Miedo de Lira y Castillo (1991), se estudia el miedo como factor central para ejercer el poder mediante la coerción y la amenaza. Se señalan como dos los factores importantes a tomar en cuenta, uno es el de legislar para legitimar la intervención militar de 1973, que penaliza retroactivamente muchas conductas que habían sido legitimas hasta el golpe militar siendo este uno de los factores de amenaza politica más potente pues son susceptibles de castigo acciones que eran legales cuando se realizaron, con esto se genera un sentimiento de inseguridad y desprotección que produce terror. El otro factor es el de que la represión politica se da en un marco de masividad y arbitrariedad de modo que cualquiera

${ }^{8}$ Kinstony Cohen 1986 Traducción D. Becker. 
se pudiera sentirse amenazado. Ambos factores apuntan a producir una sensación de impotencia y de indefensión que fragiliza a las personas, si además agregamos que se introduce la muerte como sanción posible las pautas referenciales se desarman, el desamparo se transforma en una percepción generalizada, los sujetos están inermes ante la arbitrariedad.

El querer cuestionar lo que en ese momento pasaba, como vimos era dificil, cualquier intento en ese sentido era definido como amenazante.

Ese era el contexto en el que se produce el daño, se produce una alteración del sentido que pueden tener los parámetros que evalúan la realidad. La vuelta a la democracia es una situación que no cambia en el plano del saber subjetivo substancialmente la situación. El miedo y el temor es algo que impregna el discurso en los años posteriores a la dictadura.

En el periodo del gobierno constitucional de Aylwin, y posteriormente el de Frei, se mantiene la sensación de miedo que genera el cuestionamiento a cualquier normativa, las discusiones sobre el aborto, el divorcio son una clara muestra de ello, las argumentaciones en contra de legislar sobre esas materias, giran en torno a la sanción moral, a la fantasía de la desorganización y el caos que se produciría si se legisla sobre esta materia, no se puede trasladar la discusión a un plano de argumentación in- telectual, esta se mantiene en el plano emotivo, similar al que se usa en el gobierno militar en relación al marxismo, y a cualquier discusión política.

Estas dos situaciones a las que hemos hecho referencia, la de Rosa y la del País, podemos pensarlas como historias fracturadas, quebradas, ambas necesitadas de un espacio que les posibilite su reparación. En ambas situaciones vemos que lo que falta es un leguaje que articule el relato donde los hechos puedan tener cabida, donde puedan ser nombrados, en ambas situaciones existe un temor de volver a recordar, se piensa que es inútil, que no vale la pena.

El espacio que necesita Rosa es un espacio terapéutico que le permita reconstruir, es parte borrada de la estructura psíquica ${ }^{9}$, y de esa forma reparar el daño sufrido, poder nombrar el espacio vacío que hasta ahora ha llenado con el olvido.

El espacio que nesecita el país es un espacio social que posibilite reconstruir la historia, nombrar lo que hasta ahora se ha llenado con el vacio de la amnesia.

Es muy posible que unir los pedazos no nos de un objeto igual al anterior, ni Rosa recupere lo que perdió, ni el país vuelva a ser lo que fue, es muy posible que este objeto sea distinto, pero lo que si es cierto es que no sabemos como será.

${ }^{9}$ D. Becker op. cit. 


\section{Prof.JuanaKovalskys, Coordinadoradel SeminarioPresentación de MónicaLlaña}

La Profesora Mónica Llaña, Sociologa y Magister en Educación, es docente e investigadora del Departamento de Educación, de la Universidad de Chile. Alli ha desarrollado una linea de trabajo en elárea social de sociología de la educación, en torno al tópico de la cultura escolar, considerando sus distintos actores. Su reflexión corresponde desde una lectura de los conflictos en la comunidad escolar. 